\title{
THE USE OF MULTIWAVELENGTH ARCHIVAL OBSERVATIONAL DATA FOR SCIENTIFIC DISCOVERIES: A CASE OF THE SUPERNOVA REMNANT CASSIOPEIA A
}

\author{
Dmitrijs Docenko \\ Institute of Astronomy, University of Latvia, Rainga bulv. 19, Rīga LV-1586, \\ Latvia; dima@latnet.lv
}

Received: 2012 August 9; accepted: 2012 September 14

\begin{abstract}
Most of the high-quality astronomical data after a proprietary period of typically one year are provided to open access, allowing researchers to complement their observations with the archival data in other wavelength bands, thus improving quality of the data analysis. This paper presents one example of such a use - studies of the reverse shock front passing through an oxygen-rich material in the young supernova remnant Cassiopeia A. The paper is based on the contribution to the "Baltic Applied Astroinformatics and Space Data Processing" conference, held on 2012 May 7-8 in Ventspils, Latvia.
\end{abstract}

Key words: astronomical databases: miscellaneous - supernovae: individual: Cas A

\section{INTRODUCTION}

For studies of astronomical objects, multi-wavelength or multi-epoch data are often needed. Such data are difficult to obtain from observations, as this requires use of different telescopes and differing techniques, and this increases the time from the initial proposal to the scientific results. To overcome this difficulty, virtual observatories (e.g., European Virtual Observatory ${ }^{1}$, National Virtual Observatory ${ }^{2}$ and many others) have been created and are currently of common use by astronomers. They provide the identification of and access to the archival data from many surveys and individual observations, allowing to easily overview existing information on objects and properties that are being studied. All this wealth of information is open to anyone interested to study it.

The existence of such easily accessible open data archives considerably increases the use of the wide-field and all-sky surveys, as they can later be used for many comparative observations. Also the data from many dedicated pointed observations are not analyzed enough to extract their full potential. In many cases, the data of pointed observations have been used only for a specific goal of the proposal (e.g., astrometry, or study of a single object out of many in the field of view). Such archival data may be successfully used for a new research and discoveries. The possibility to combine the data from different wavelength regions is especially

\footnotetext{
1 Available on the Internet at http://www.euro-vo.org

2 Available on the Internet at http://www.us-vo.org/
} 
important.

The goal of this paper is to show an example of the use of open-access archival data for astrophysical studies of the young supernova remnant Cassiopeia A (Cas A). This object has been extensively studied in radio (e.g., Baars et al. 1977), submillimeter (e.g., Dunne et al. 2003), infrared (e.g., Rho et al. 2008), optical (e.g., Reed et al. 1995), X-ray (e.g., Hughes et al. 2000) and gamma-ray (e.g., Iyudin et al. 1994) ranges.

Cas A is a young (age of about $340 \mathrm{yr}$ ) shell-type remnant of a type IIb supernova located near Galactic plane at a distance about $3.4 \mathrm{kpc}$. It is one of the brightest sources in the radio sky, a very bright X-ray source, but its visible emission is relatively weak. Its optical and near-infrared spectrum is dominated by emission lines from the metal-dominated emission knots being crossed by the reverse shock, whereas far-infrared to sub-millimeter spectrum is dominated by a warm and cold dust emission (e.g., Barlow et al. 2010).

In this paper, we review various types of the archival observational data and show how they may be combined for a successful scientific analysis.

\section{TYPES OF ARCHIVAL OBSERVATION DATA}

The archival observations may be of different processing level, determining an effort which should be put into creating the results of scientific quality from the data. Usually, "Level 0" denotes the raw uncalibrated instrumental data, and progressively higher level numbers denote the data which are pre-processed (bad pixels, hot pixels, glitches, etc. removed) and processed (converted from instrumental to physical units, e.g., from electrons per pixel to $\mathrm{W} / \mathrm{cm}^{2} / \mathrm{nm} / \mathrm{sr}$ ).

Here is the list of examples of the data types of the archival data.

Light curve. This is a one-dimensional dependence of intensity from time in some spectral filter integrated over certain angular aperture.

Spectrum. This is an one-dimensional distribution of intensity over wavelength (or, equivalently, energy) integrated over a certain angular aperture.

Photometric map. This is a two-dimensional distribution of intensity in some spectral filter over certain celestial region.

Data cube. This is a three-dimensional data representation, combining properties of a spectrum and a map. For each pixel of the map, a spectrum is available. Care should be taken in analysis of the spectra from this data type, as point spread function of a diffraction-limited instrument is dependent on the wavelength. This data type is typical for modern infrared instruments, such as the IRS instrument of the Spitzer Space Telescope and the PACS instrument of the Herschel Space Observatory.

Event list. In this data type, usually for the X-ray telescopes operating in the photon counting mode, for each detected photon its arrival time, energy and position are stored. The result is a one-dimensional sequence of "events" which represents however a four-dimensional distribution over these parameters. This data type enables to obtain (1) an object spectrum by selecting angular aperture and summing over time, (2) an object map by summing over energies and time, or (3) a light curve by summing over energy.

The images of the Cas A obtained from different data types are presented on Figures $1-4$.

To be compared directly, the observation data from different sources should be 


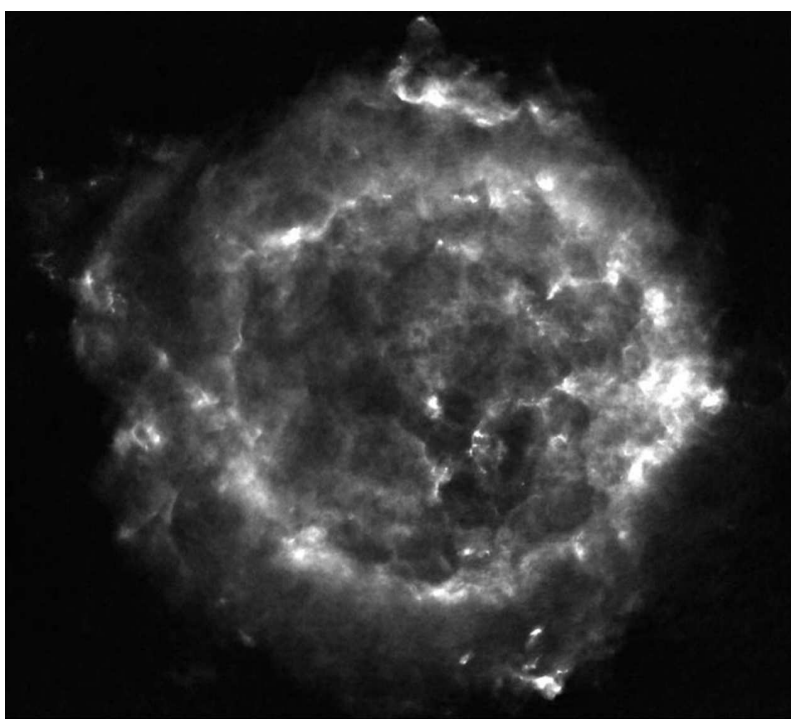

Fig. 1. An example of processing the raw observation data. To obtain this $5 \mathrm{GHz}$ radio brightness map of the Cas A (ObsID AR0435), the raw archival data from different configurations of the Very Large Array (VLA) radio telescopes had to be combined and correlated, bad data detected and removed, then a source model had to be created using the CLEAN algorithm. This requires detailed understanding of the telescope operation and the data processing.

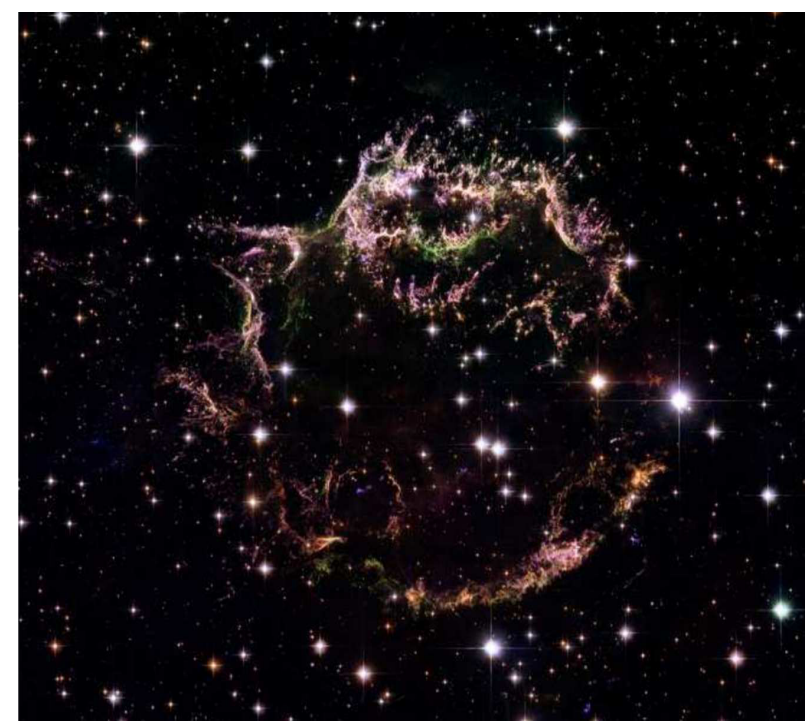

Fig. 2. An example of the processed photometric map. This Hubble Space Telescope (HST) image of the Cas A (proposal 10286) was obtained in several photometric bands in December of 2004. Several simple steps are enough to convert the instrumental units of the image to the physical units. 


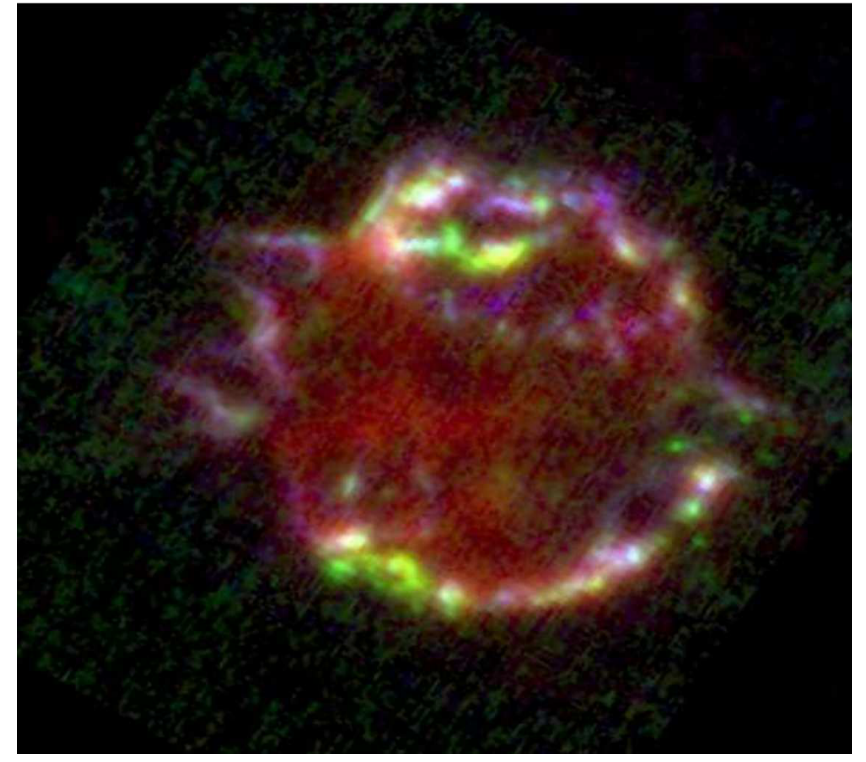

Fig. 3. An example of the data projected out from the data cube (spectral map). This Spitzer Space Telescope image (Program Id 3310) was created from a data cube by selecting certain wavelength regions for the image colors (red - [O IV] $25.91 \mu \mathrm{m}$, green [Ne II] $12.81 \mu \mathrm{m}$, blue - [Ar II] $6.985 \mu \mathrm{m}$ line emission).

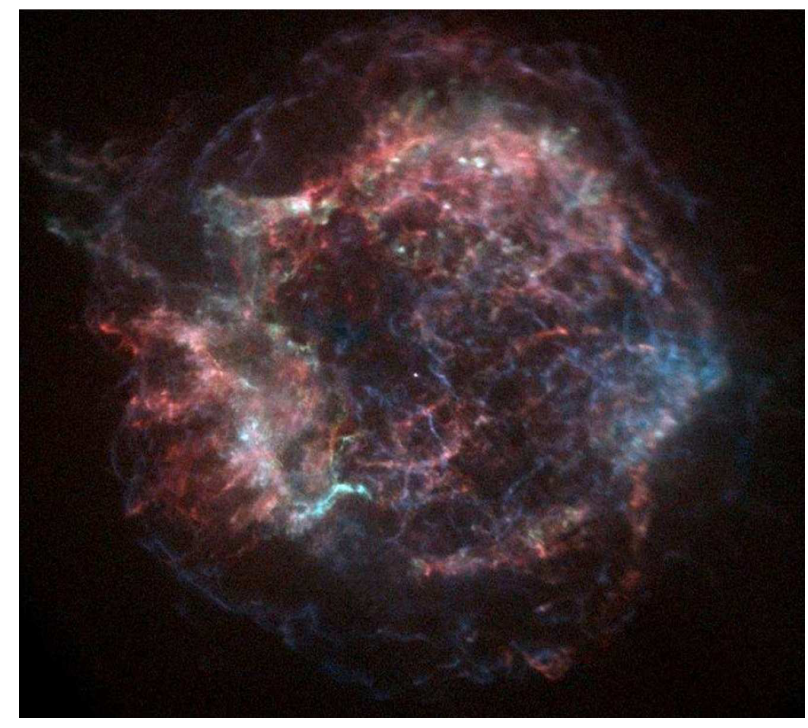

Fig. 4. An example of the data created from the event list. This Chandra X-ray Observatory image (ObsID 4638) was created by summing up all events for each image pixel observed during exposures in certain energy ranges (red $-0.5-1.6 \mathrm{keV}$, green $1.6-2.2 \mathrm{keV}$, blue $-2.2-5.0 \mathrm{keV})$. 
converted to the same type and binned to the same angular regions with the same point spread function (PSF). As typically all the data are present in the FITS $^{3}$ This processing usually includes rebinning to fine sub-pixels to exclude angular resolution worsening in the further processing, transformation to the common coordinate system, convolution to the same angular resolution (or, rather, to the same PSF), and finally rebinning to the same pixel size. Functions to perform these operations are either contained in the CFITSIO library, or may be easily constructed using functionality of this library.

\section{ARCHIVAL OBSERVATIONS OF THE CASSIOPEIA A}

The high-quality observations of the Cas A, illustrated on Figures 1-4, were performed for specific purposes of studying kinematics and chemistry of the ejecta, dust formation and destruction in the supernova, electron acceleration on the shock front, supernova remnant evolution, etc. However, these sets of data, when analyzed jointly, allow also other types of scientific analysis.

In our paper (Docenko \& Sunyaev 2010), we have studied jointly the observational data from visible, near-, mid- and far-infrared spectroscopy to infer the structure of the reverse shock passing through the dense oxygen-dominated ejecta knots. This became possible only thanks to analysis of the data from all of these wavelength ranges. For example, the absence of the far-infrared [O I] $145.5 \mu \mathrm{m}$ line allowed us to exclude electron densities below $2 \times 10^{5} \mathrm{~cm}^{-3}$ in the post-shock photo-ionized region prior to the shock-induced cloud disruption, and the combination of near-infrared and mid-infrared [Fe II] lines at $1.3209 \mu \mathrm{m}$ and 17.94 $\mu \mathrm{m}$ allowed then to determine exactly its mean temperature to be about $1750 \mathrm{~K}$. Relative intensities of spectral lines allowed us to recover chemical composition, physical conditions and geometrical structure of the Cas A supernova remnant reverse shock.

\section{SUMMARY AND CONCLUSIONS}

The use of the archival data sometimes may be sufficient to test the new ideas about the mechanism of certain astrophysical processes. If the new observations are made, archival data can be used for comparison and, in many cases, for gaining additional insight into the processes occurring in the astrophysical object under study. At present, this task is facilitated considerably by the use of virtual observatories and is becoming a standard way in astronomer's work. The paper summarizes various processing levels and types of the archival data and shows their application on the example of the Cas A young supernova remnant.

It is shown that the combination of multi-wavelength data of Cas A obtained in different observational campaigns allows performing a scientific analysis (in this case, determination of the structure of the reverse shock passing through a dense oxygen-dominated ejecta knots) which cannot be performed on the data from any single observational campaign.

3 FITS stands for "Flexible Image Transport System" and is the standard astronomical data format endorsed by both NASA and the IAU. Popular graphical software for a work with the FITS files includes fv, graphical FITS file viewer and editor, and ds9, an astronomical imaging and data visualization application. Many useful functions for editing FITS files are contained in the CFITSIO Software Library available in C and Fortran. 
ACKNOWLEDGMENTS. The author is thankful to the organizers of the BAASP 2012 conference - Valdis Avotiņš and Liene Riekstinsa - for the invitation and hospitality.

\section{REFERENCES}

Baars J. W. M., Genzel R., Pauliny-Toth I. I. K., Witzel A. 1977, A\&A, 61, 99

Barlow M. J., Krause O., Swinyard B. M. et al. 2010, A\&A, 518, L138

Docenko D., Sunyaev R. A. 2010, A\&A, 518, A59

Dunne L., Eales S., Ivison R. et al. 2003, Nature, 424, 285

Hughes J. P., Rakowski C. E., Burrows D. N., Slane P. O. 2000, ApJ, 528, L109

Iyudin A. F., Diehl R., Bloemen H. et al. 1994, A\&A, 284, L1

Reed J. E., Hester J. J., Fabian A. C., Winkler P. F. 1995, ApJ, 440, 706

Rho J., Kozasa T., Reach W. T. et al. 2008, ApJ, 673, 271 